\title{
Ações coletivas para o controle do diabetes mellitus tipo 2 na Atenção Primária à Saúde: Um relato de experiência
}

Collective actions for the control of type 2 diabetes mellitus in Primary Healthcare: An experience report

Acciones colectivas para el control de la diabetes mellitus tipo 2 en Atención Primaria de Salud: Informe de experiencia

Letícia Mayra de Oliveira Manoel ORCID: https://orcid.org/0000-0002-9391-0841 Universidade de São Paulo, Brasil E-mail: leticiamayra.farma@gmail.com Lucas Gaspar Ribeiro ORCID: https://orcid.org/0000-0003-4842-4294 Universidade de Ribeirão Preto, Brasil E-mail: lucasgasparribeiro@gmail.com

Rinaldo Eduardo Machado de Oliveira ORCID: https://orcid.org/0000-0003-1684-1456 Universidade de São Paulo, Brasil E-mail: rinaldo.eduardo@usp.br Julieta Mieko Ueta

ORCID: https://orcid.org/0000-0003-2645-0432 Universidade de São Paulo, Brasil E-mail: julietapurame@gmail.com

\begin{abstract}
Resumo
O objetivo deste estudo foi relatar a experiência da realização de atendimentos coletivos para pessoas com diabetes mellitus tipo 2 em uma Unidade de Saúde da Família do distrito sanitário oeste do município de Ribeirão Preto, São Paulo. Trata-se de um relato de experiência construído a partir do escopo de pesquisa qualitativa de característica pesquisa-ação. A coleta de dados ocorreu entre agosto e outubro de 2018, e teve por finalidade identificar e caracterizar as pessoas com diabetes quanto à necessidade de inclusão ao grupo de intervenção para atendimentos coletivos. Verificou-se 126 pessoas com diabetes mellitus tipo 2, sendo que 70,6\% eram do sexo feminino, média de idade de 59,7 anos, 72,2\% possuíam diagnóstico concomitante de hipertensão arterial sistêmica, 31,7\% apresentavam atraso na frequência de consultas médicas ou exames laboratoriais e 17,9\% apresentavam hemoglobina glicada acima de $8 \%$ no último exame realizado. Foram convidadas 40 pessoas e compareceram $60 \%$ delas para participação nas ações coletivas destinadas ao controle do diabetes. No decorrer dos encontros foram abordados temas de educação em diabetes, atualizações nas prescrições de medicamentos e solicitações de exames laboratoriais. Acredita-se que a estratégia utilizada nesta intervenção possa ser reproduzida em outros serviços da Atenção Primária à Saúde a fim de garantir a integralidade do cuidado às pessoas com diabetes.
\end{abstract}

Palavras-chave: Atenção primária à saúde; Diabetes Mellitus Tipo 2; Controle glicêmico.

\begin{abstract}
This study aimed to report the experience of providing collective care for people with type 2 diabetes mellitus in a Family Healthcare Unit in the western sanitary district of Ribeirão Preto, São Paulo. It is an experience report built from the scope of qualitative research of characteristic research-action. Data collection took place between August and October 2018 and aimed to identify and characterize people with diabetes regarding the need for inclusion in the intervention group for collective care. It was found 126 people with type 2 diabetes mellitus, of which $70.6 \%$ were female, mean age 59.7 years, $72.2 \%$ had a concomitant diagnosis of systemic arterial hypertension, $31.7 \%$ had a delay in the frequency of medical appointments or laboratory tests and $17.9 \%$ had glycated hemoglobin above $8 \%$ in the last test performed. Forty people were invited and $60 \%$ of them attended to participate in collective actions aimed at controlling diabetes. During the meetings, topics of diabetes education, updates on medication prescriptions and requests for laboratory tests were addressed. It is believed that the strategy used in this intervention can be reproduced in other Primary Health Care services to ensure comprehensive care for people with diabetes.
\end{abstract}

Keywords: Primary health care; Diabetes Mellitus, Type 2; Glycemic control. 


\begin{abstract}
Resumen
El objetivo de este estudio fue reportar la experiencia de brindar atención colectiva a personas con diabetes mellitus tipo 2 en una Unidad de Salud de la Familia en el distrito sanitario occidental de Ribeirão Preto, São Paulo. Es un informe de experiencia construido a partir del alcance de la investigación cualitativa de investigación-acción característica. La recolección de datos se llevó a cabo entre agosto y octubre de 2018, y tuvo como objetivo identificar y caracterizar a las personas con diabetes en cuanto a la necesidad de inclusión en el grupo de intervención para la atención colectiva. Se encontró que 126 personas con diabetes mellitus tipo 2, de las cuales 70,6\% eran mujeres, edad média 59,7 años, 72,2\% tenían diagnóstico concomitante de hipertensión arterial sistémica, 31,7\% presentaban retraso en la frecuencia de citas médicas o pruebas de laboratorio y el 17,9\% tenía hemoglobina glucosilada superior al $8 \%$ en la última prueba realizada. Se invitó a cuarenta personas y el $60 \%$ de ellas asistió para participar en acciones colectivas dirigidas al control de la diabetes. Durante las reuniones, se abordaron temas de educación diabética, actualizaciones sobre prescripciones de medicamentos y solicitudes de pruebas de laboratorio. Se cree que la estrategia empleada en está intervención puede reproducirse en otros servicios de Atención Primaria de Salud con el fin de garantizar una atención integral a las personas con diabetes.
\end{abstract}

Palabras clave: Atención primaria de salud; Diabetes Mellitus Tipo 2; Control glucémico.

\title{
1. Introdução
}

O diabetes mellitus tipo 2 (DM2) é um distúrbio metabólico que tem como principal característica a manutenção da hiperglicemia no decorrer de um longo período. Pode ser atribuído à deficiência na produção ou na ação da insulina, mas também à associação destes dois fatores (Sociedade Brasileira de Diabetes, 2019). Estudos recentes apontam para a existência de diversos subtipos de diabetes, e o conhecimento e estratificação de tais fenótipos e genótipos possibilitarão tratamentos mais individualizados (Skyler, et al., 2017).

O DM2 é responsável por $90 \%$ a $95 \%$ dos casos de diabetes e decorre principalmente da adoção de estilo de vida inadequado, combinação de má alimentação, sedentarismo, tabagismo, etilismo, além de ser um dos efeitos do envelhecimento populacional (Torres, et al., 2018).

O Brasil ocupa a quarta posição entre os países com maior número de pessoas convivendo com a doença, somando cerca de 14,3 milhões de casos e estando atrás apenas da China, Índia e dos Estados Unidos da América (Borges \& Lacerda, 2018). A última Pesquisa Nacional de Saúde estimou que 7,7\% das pessoas com idade igual ou superior a 18 anos tinham relatado diagnóstico médico de diabetes no ano de 2019 (Instituto Brasileiro de Geografia e Estatística, 2019). Dados municipais mostraram que em Ribeirão Preto, São Paulo (SP) o cenário é ainda mais preocupante, com números próximos a $15 \%$ da população (Moraes, et al., 2010).

O diabetes está relacionado a diversas complicações, principalmente micro e macrovasculares. O controle inadequado é responsável pelo alto grau de morbimortalidade associado à doença (American Diabetes Association, 2021). Além de todos os desfechos negativos decorrentes das complicações, que resultam em perda da qualidade de vida e morte precoce, destaca-se o elevado custo financeiro que o diabetes acarreta aos sistemas de saúde, sendo responsável por $12 \%$ dos gastos globais em saúde (Zimmet, et al., 2016).

A oferta de serviços de saúde de forma fragmentada e com ações reativas e episódicas, voltadas prioritariamente a assistir quadros de agudização das condições crônicas, representam um infortúnio no manejo dessas doenças, com ênfase no diabetes (Mendes, 2012, p. 21). Para evitar o exposto, as práticas relacionadas à educação em saúde e ao autocuidado, principalmente medidas não farmacológicas, são cruciais para o controle da condição (Campbell, Willians \& Egede, 2021). As palestras e transmissão de conhecimento são as principais estratégias coletivas empregadas no Brasil. Contudo, esta abordagem mostra-se ineficaz, com baixo impacto na mudança comportamental e melhorias do autocuidado (Gomes, Silva, Pereira \& Rabelo, 2019).

Neste sentido, e considerando a necessidade da mudança nos modelos de atenção à saúde, pressupõe-se a imprescindibilidade da adoção de programas centrados na instrumentalização do sujeito na tomada de decisões, na 
responsabilização pelos seus cuidados em saúde e controle da condição, e pautado na prevenção das complicações crônicas (Torres, et al., 2018).

Para a mudança de tal característica, uma estratégia bastante efetiva no cuidado às pessoas com condições crônicas não transmissíveis, como o diabetes, é o atendimento coletivo por equipe multiprofissional (Torres, et al., 2018). Esta modalidade assistencial difere do grupo tradicional, pois estimula a participação ativa do usuário, construindo em conjunto objetivos claros e adequados a realidade local, com ampliação do acesso, fortalecimento da adesão ao tratamento, apoio ao autocuidado e a mudança de hábitos, bem como, o aumento da satisfação da equipe e pacientes e melhoria dos desfechos clínicos (Ferreira \& Kind, 2010). Além disso, configura-se um espaço no qual podem ser realizadas atualizações de receitas, solicitações de exames laboratoriais, bem como discussões sobre os resultados de exames e terapia medicamentosa. Desta maneira, criamos um espaço no qual o indivíduo utiliza os princípios de diálogo, participação, problematização e ação, para favorecer a formação da consciência crítica (Ancioli, 2021).

A Atenção Primária à Saúde (APS) e a estratégia de saúde da família (ESF), representam um cenário favorável para a implantação de tais práticas, uma vez que trabalham com o território adscrito e têm por base as ações de promoção, prevenção, tratamento, reabilitação e atenção à saúde. Assim, torna-se possível a compreensão da dinâmica dos lugares e indivíduos, desigualdades sociais, iniquidades em saúde, bem como, o estabelecimento de relações de vínculo, afetividade e confiança entre as equipes e usuários. Isso, garante um elevado grau de resolutividade das ações e a longitudinalidade do cuidado (Figueiredo, 2012).

Na utilização de estratégias como o atendimento coletivo na APS é imprescindível que as ações sejam pautadas no planejamento em saúde (Faria, Campos, Santos, 2018). Ao se apropriar do planejamento estratégico situacional, consideramse: identificação dos problemas de saúde, classificação e priorização, descrição e explicação do problema, seleção e delineamento dos "nós críticos", desenho da operação, identificação dos recursos necessários e viabilidade do plano, elaboração e execução do plano operativo e gestão do plano com reavaliações periódicas (Faria, Campos \& Santos, 2018). Utilizando este modelo idealiza-se um modelo ou estratégia, como o atendimento coletivo ao diabetes, de maneira mais racional e condizente com as necessidades da população adscrita, levando em consideração as peculiaridades locais e complexidades do processo (Fontana, et al., 2020). Neste sentido, o objetivo deste artigo é relatar a experiência da realização de atendimentos coletivos para pessoas com DM2 em uma Unidade de Saúde da Família do distrito sanitário oeste do município de Ribeirão Preto, SP.

\section{Metodologia}

O desenho metodológico das intervenções coletivas foi construído a partir do escopo de pesquisa qualitativa de característica pesquisa-ação (Gil, 2002). A atividade foi desenvolvida no cenário de atuação da Residência Multiprofissional em Atenção Integral à Saúde da Faculdade de Medicina de Ribeirão Preto da Universidade de São Paulo (FMRP-USP), que conta com profissionais de diversas áreas, apresentando assim oportunidades de atuação conjunta para o cuidado das pessoas com doenças crônicas não transmissíveis.

A coordenação da atividade ficou a cargo da farmacêutica residente do programa vinculada à unidade e auxiliada pela equipe fixa e residentes de outras áreas (nutrição, odontologia, terapia ocupacional, fonoaudiologia e fisioterapia). Destaca-se a colaboração do médico responsável pela unidade nos atendimentos coletivos.

O projeto inicial que deu origem ao presente trabalho é baseado na caracterização sociodemográfica, clínica e da utilização de medicamentos de pessoas com diabetes e hipertensão arterial sistêmica (HAS) pertencentes a seis unidades de saúde da família do distrito oeste de Ribeirão Preto. Justifica-se pelos elevados índices de prevalência do DM2 no município e fragmentação do cuidado. Embora tais características sugiram um controle inadequado da doença, um estudo realizado no município indicou que a maioria dos idosos acompanhados pela APS e com esquema farmacoterapêutico apropriado, 
apresentam resultados de hemoglobina glicada inferior de $8 \%$ (Oliveira \& Franco, 2021). Desta maneira, um cuidado qualificado pode garantir desfechos positivos no controle do diabetes. A partir disso foi proposto um recorte na tentativa de realizar um projeto de intervenção em uma destas unidades, que posteriormente poderá ser aprimorado e ampliado às demais unidades.

O foco deste trabalho foi a educação em saúde de pessoas com DM2, bem como a qualificação do cuidado deste grupo. As intervenções propostas foram baseadas no planejamento estratégico situacional em saúde e as etapas do plano operativo encontram-se descritas na Figura 1.

Figura 1. Etapas do planejamento para o desenvolvimento dos atendimentos coletivos em diabetes. Unidade de Saúde da Família, Ribeirão Preto, São Paulo, 2018.

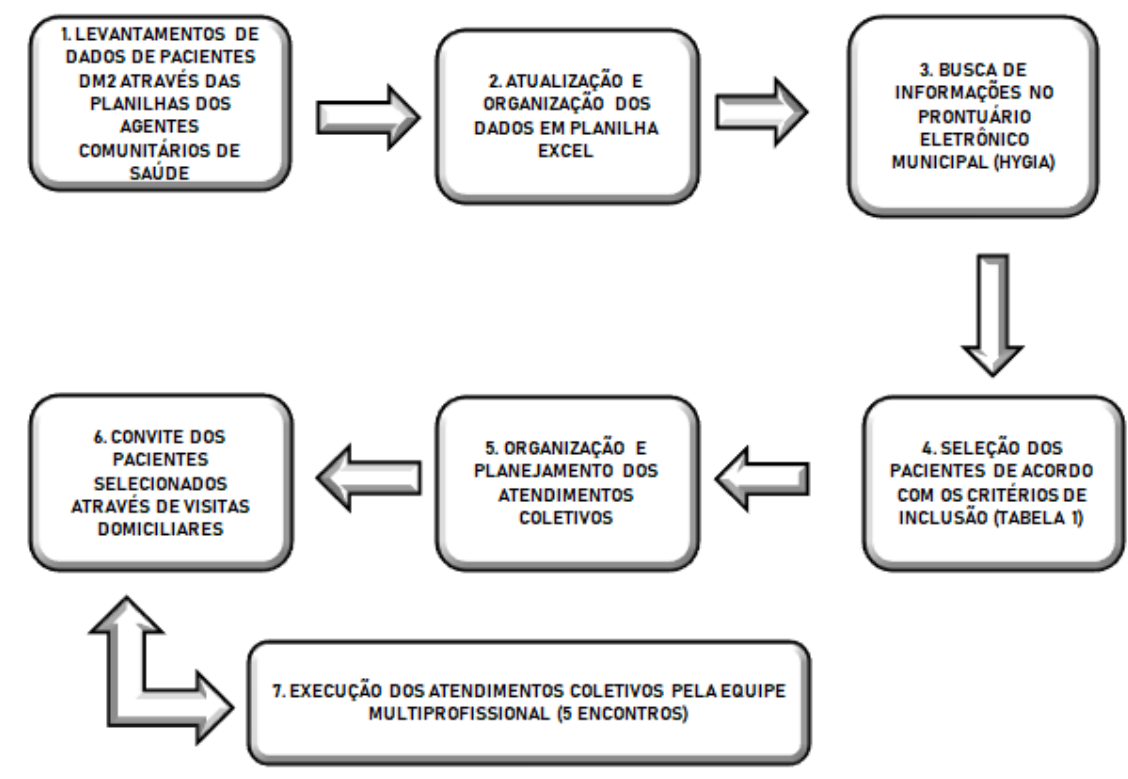

Fonte: Autores.

Etapa 1: Levantamento de dados: ocorreu no período de agosto a outubro de 2018, com o objetivo de identificar as pessoas com diabetes e hipertensão arterial sistêmica (HAS) que estavam em seguimento na USF, bem como o endereço, telefones e dados cadastrais. Para tal, foram utilizados os dados disponibilizados por Agentes Comunitários de Saúde (ACSs).

Etapa 2: Atualização dos dados: foram realizadas inclusões, exclusões e atualizações de dados de acordo com a necessidade.

Etapa 3: Rede eletrônica municipal: o município conta com o Hygia, um sistema de informatização da saúde no qual ficam registrados dados cadastrais dos usuários e da utilização dos serviços de saúde do município vinculados ao sistema. Também possibilita o acesso a exames laboratoriais e prescrições. Assim, realizou-se um levantamento do resultado e data do último exame de hemoglobina glicada (HbA1c), bem como, a data da última consulta na atenção básica de todas as pessoas com diabetes cadastradas na USF.

Etapa 4: Seleção das pessoas: foram selecionadas todas as pessoas com diabetes cuja data da última consulta era superior a seis meses ou último resultado do exame de HbA1c maior que $8 \%$. A equipe teve autonomia para definir a entrada de pessoas por outros critérios que julgasse necessário.

Etapa 5: Organização da atividade: ficou definido pela equipe que seriam realizados cinco encontros sequenciais e semanais entre novembro e dezembro de 2018. 
Etapa 6: Convite aos usuários: foram elaborados e impressos os convites com a data e breve descrição dos encontros. A farmacêutica, juntamente com os ACSs, realizou a entrega dos convites. Neste momento forneceram-se explicações detalhadas acerca dos atendimentos coletivos. Aqueles que não se encontravam no domicílio foi deixado o convite na caixa de correio e realizado contato telefônico por até três vezes.

Etapa 7: Execução da atividade: de acordo com a definição da equipe multiprofissional, os encontros foram organizados da seguinte maneira: o primeiro encontro foi para apresentação da proposta e integração dos usuários com a equipe. Nesta ocasião foram realizadas solicitações de exames laboratoriais e atualizações de prescrições após avaliação crítica da necessidade (validade da prescrição para no máximo um mês). Posteriormente foi proposto o levantamento das principais dúvidas em relação ao convívio com o diabetes para nortear os encontros subsequentes. Pactuou-se o tema: funcionamento do organismo e diabetes para o primeiro encontro.

Os três encontros subsequentes aborfou-se de maneira dinâmica e participativa os temas levantados pelos usuários, baseando a fala na tríade de controle do diabetes: alimentação, exercício físico e terapia medicamentosa, além de trabalhar as demandas suscitadas por eles.

O último encontro teve como foco o encerramento das atividades e compreensão sobre a percepção dos participantes em relação ao grupo. Para isso foi proposta a realização de uma roda de conversa e avaliação por palavra-chave. Ao final do último encontro foi oferecido um lanche da tarde saudável para o encerramento das atividades.

Os materiais utilizados nas atividades foram os mapas de conversação em diabetes (Federação Internacional de Diabetes [IDF] ), concedidos pela Secretaria Municipal de Saúde de Ribeirão Preto, SP, protótipos de alimentos (fashion cook) - material permanente da residência multiprofissional em saúde - além dos resultados de exames laboratoriais, prescrições e exame clínico (antropométrico e sinais vitais) dos próprios usuários para discutirmos em conjunto, a fim de suscitar a interação e participação.

Detalhes das definições de cada encontro podem ser visualizados no Quadro 1.

Quadro 1. Detalhamento da dinâmica dos encontros. Unidade de Saúde da Família, Ribeirão Preto, São Paulo, 2018.

\begin{tabular}{|c|l|}
\hline \multirow{2}{*}{$1^{\text {o }}$ Encontro } & $\begin{array}{l}\text { Apresentação da proposta. } \\
\text { Levantamento das principais dúvidas. } \\
\text { Solicitação de exames e atualização das prescrições médicas (curto período). } \\
\text { Abordagem do tema: como o organismo e o diabetes funcionam, aplicando o mapa } \\
1 \text { de conversação em diabetes. }\end{array}$ \\
\hline \multirow{2}{*}{$2^{\circ}$ Encontro } & $\begin{array}{l}\text { Discussão de resultados de exames com os usuários (curto período). } \\
\text { Abordagem do tema: convivendo com o diagnóstico e prevenindo complicações } \\
\text { crônicas. Aplicação o mapa 6 de conversação em diabetes. Envolvimento do núcleo } \\
\text { familiar no enfrentamento do diabetes. }\end{array}$ \\
\hline $3^{\circ}$ Encontro & $\begin{array}{l}\text { Abordagem do tema: alimentação saudável para o bom controle do diabetes: } \\
\text { dinâmica interativa utilizando o fashion cook. }\end{array}$ \\
\hline $4^{\circ}$ Encontro & $\begin{array}{l}\text { Abordagem do tema: atividade física versus exercício físico versus sedentarismo. } \\
\text { usuários. }\end{array}$ \\
\hline $5^{\circ}$ Encontro & $\begin{array}{l}\text { Avaliação da percepção dos pacientes em relação ao grupo através de palavras- } \\
\text { chave. }\end{array}$ \\
\hline & Estabelecimento de metas em curto prazo. \\
\hline
\end{tabular}




\section{Resultados e Discussão}

Os resultados serão expostos em dois grupos sequenciais. O primeiro grupo são os dados de obtenção das informações e convite dos participantes (Etapas 1 a 7 da Figura 1). O segundo grupo traz o desenvolvimento detalhado das atividades, desde a proposta metodológica até os resultados de cada encontro. A Tabela 1 mostra o perfil da população cadastrada na USF.

Tabela 1. Características sociodemográficas e clínicas da população cadastrada. Unidade de Saúde da Família. Ribeirão Preto, São Paulo, 2018. (N=2391).

\begin{tabular}{lcc}
\hline Variável & N & \% \\
\hline Idade & & \\
$<60$ anos & 2065 & 86,4 \\
$\geq 60$ anos & 326 & 13,6 \\
Diagnóstico de diabetes mellitus tipo 2 & 126 & 5,2 \\
Diagnóstico de hipertensão arterial sistêmica & 268 & 11,2 \\
Diagnóstico de diabetes e hipertensão arterial & 91 & 3,8 \\
sistêmica concomitantes & & \\
\hline
\end{tabular}

Fonte: Prontuários dos pacientes e Sistema de Informação Municipal Hygia.

Nesta USF, verifica-se baixa frequência de pessoas idosas, sendo a população composta majoritariamente por adultos jovens. Além disso, a prevalência estimada de diabetes foi de 5,2\%. Estudos apontaram que, no município de Ribeirão Preto, a prevalência do diabetes é de 15\% (Moraes, Freitas, Gimenos \& Modini, 2010). Acredita-se que tal discrepância é multifatorial e pode relacionar-se com a existência de cobertura da ESF há mais de vinte anos com a qualificação do cuidado em saúde da população. Além disso, pode-se justificar este achado com a característica demográfica da população, composta majoritariamente por crianças e adultos jovens. A Tabela 2 descreve o perfil das pessoas com DM2.

Tabela 2. Características sociodemográficas e clínicas das pessoas com diabetes mellitus tipo 2. Unidade de Saúde da Família. Ribeirão Preto, São Paulo, 2018. (n=126).

\begin{tabular}{lcc}
\hline Variável & $\mathbf{n}$ & \% \\
\hline Sexo & 89 & 70,6 \\
Feminino & 37 & 29,4 \\
Masculino & 59,7 & 17,5 \\
Média de idade (anos) & 22 & 31,7 \\
Hemoglobina glicada $\geq 8$ & 40 & \\
Consultas e exames atrasados & & \\
\hline
\end{tabular}

Fonte: Prontuários físicos e Sistema de Informação Municipal Hygia.

Nesta USF observou-se maior frequência de diabetes nas mulheres. Isso pode ser justificado pelo fato que os homens têm maior dificuldade em procurar os serviços de saúde, ao passo que as mulheres buscam com maior frequência o atendimento médico para os cuidados em saúde. (Moura, Gomes \& Pereira, 2017) A média de idade das pessoas com diabetes foi de 59,7 anos e, embora a maior frequência de diabetes seja após os 60 anos, pode-se vincular este achado as características etárias da população estudada (Brasil, 2017).

Dentre as 126 pessoas com o diagnóstico, 91 possuíam concomitantemente Hipertensão Arterial Sistêmica (72,2\%), e é de grande importância o bom controle da pressão arterial em pessoas com diabetes a fim de reduzir o risco de eventos 
cardiovasculares. A American Diabetes Association recomenda que adultos com diabetes e hipertensão devam ter uma pressão arterial alvo menor que140/90 mmHg e que a individualização baseada em risco para metas menores, como 130/80 mmHg, pode ser apropriada para algumas pessoas.

Evidenciou-se que 31,7\% das pessoas com diabetes nesta USF, apresentavam atraso na frequência de consultas de rotinas ou exames laboratoriais. De acordo com o preconizado no protocolo municipal de hipertensão e diabetes as recomendações são correlacionadas com o risco cardiovascular do indivíduo, sendo que aqueles com baixo risco deveria ser acompanhados, no mínimo a cada seis meses, os de risco intermediário no mínimo a cada três meses, e os de alto risco a cada dois meses (Ribeirão Preto, 2018). Desta forma, considerou-se consulta atrasada todas com data superior a seis meses para englobar todos os grupos.

Além disso, 17,9\% apresentavam último exame de HbA1c acima de 8\%. Empregou-se o valor de HbA1c superior a $8 \%$ para indicar controle inadequado do diabetes. No entanto é importante ressaltar que embora as metas terapêuticas sejam em torno de 7\% nos adultos e entre 7,5\% e 8,5\% nos idosos, estas devem ser individualizadas e avaliadas caso a caso. Desta forma, a Sociedade Brasileira de Diabetes (2019) assumiu um controle mais flexível aproximando-se de 8,5\% para pacientes com menos motivação para o tratamento, maior risco de hipoglicemia, duração longa da doença, com idade mais avançada e menor expectativa de vida, presença de outras doenças, ou complicações macrovasculares.

Com esses dados e utilizando os critérios de inclusão do grupo, foram selecionadas e identificadas sessenta e duas pessoas com perfil para participar dos atendimentos coletivos, sendo quarenta com consulta atrasada e vinte e duas com $\mathrm{HbA} 1 \mathrm{c}$ alterada. Foram convidadas quarenta pessoas das sessenta e duas selecionadas, pois não foi possível localizar algumas, mesmo após ao menos uma tentativa de entrega por visita domiciliar e três tentativas via telefone. Também foram excluídas as pessoas que eram impossibilitadas de se deslocar ou participar das discussões (acamados e pessoas com déficits cognitivos). Ao final, compareceram $60 \%$ das pessoas convidadas.

Dentre os vinte e quatro participantes dos atendimentos coletivos, oito possuíam HbA1c acima de 8\%, sendo a média deste exame de $10,03 \%$. Os outros dezesseis pacientes que compareceram, embora apresentassem HbAlc inferior a $8 \%$ estavam com a rotina de consultas/exames laboratoriais atrasada. Desta forma não foi possível identificar com exatidão o número de pessoas com diabetes e HbAlc menor que 8\%, devido a inexistência de alguns exames no primeiro encontro. No total, foram solicitados treze pedidos de exames laboratoriais acompanhadas de agendamentos para posterior retorno individual. Foram ainda atualizadas dez receitas após avaliação crítica da demanda e discussão multiprofissional. As prescrições foram disponibilizadas para um período máximo de um mês, a fim de controlar o retorno dessas pessoas para acompanhamento.

Além da solicitação de exames e receitas, os encontros objetivaram trabalhar assuntos de educação em diabetes a partir dos temas levantados pelos próprios pacientes no primeiro encontro. As principais demandas apresentadas foram respectivamente: alimentação saudável para o controle do diabetes, terapia medicamentosa, envolvimento da família para apoio no cuidado e utilização de terapias complementares como chás, plantas medicinais e alimentos terapêuticos.

Conforme exposto anteriormente, no primeiro encontro realizou-se a apresentação da proposta, solicitação de exames laboratoriais, atualização de prescrições, levantamento das principais dúvidas e abordagem do tema: como o corpo e o diabetes funcionam. Para tal foi utilizando o mapa 1 de conversação em diabetes (IDF, 2019). Este mapa apresenta de forma lúdica e interativa a fisiopatologia do diabetes, comparando o corpo de um indivíduo saudável ao do diabético, utilizando uma fábrica como modelo, visando a melhor compreensão do usuário.

Os participantes foram dispostos em roda e o mapa foi posicionado ao centro para melhor visualização, o facilitador auxiliou com perguntas disparadoras e incitou a discussão entre o grupo. Durante a discussão observou-se dúvidas em relação a fisiopatologia do DM2, desafios de alcançar mudanças concretas de hábitos de vida e complicações do diabetes. Houve intensa 
participação e interação, com questionamentos e trocas de experiências. Quando necessário, o facilitador realizou intervenções e esclareceu dúvidas.

No segundo encontro foi realizada uma breve discussão sobre exames laboratoriais solicitados para seguimento, salientando a importância que o controle e acompanhamento do diabetes tem na instrumentalização da equipe de saúde para auxiliar no manejo do cuidado. Exemplificado com os resultados de um dos usuários não identificado para o grupo que apresentava glicemia de jejum de $120 \mathrm{mg} / \mathrm{dl}$ e $\mathrm{HbA} 1 \mathrm{c}$ de $12,4 \%$ e perguntado ao grupo o que estes exames e os valores representavam, permitiu-se que discutissem entre si e realizassem comentários.

Nesta etapa os participantes puderam discutir entre si e com o facilitador, sendo que ao final da discussão este explicou o significado dos exames e resultados. Ressaltou-se que HbA1c revela um filme de como tem se mantido a glicemia ao longo dos últimos três meses, e a glicemia de jejum é uma foto do momento do exame, podendo não refletir um bom controle do diabetes mesmo estando na faixa adequada. Enfatizou-se ainda que as metas devem ser pactuadas individualmente com a equipe de saúde.

Aproveitando a temática de exames, foi exposto as complicações crônicas que o controle glicêmico inadequado pode provocar a longo prazo. Utilizado como ferramenta o mapa 6 de conversação: entendendo os muitos fatores de controle do diabetes (IDF, 2019). Este mapa expõe as problemáticas das complicações micro e macrovasculares através de um vulcão em erupção numa ilha, a qual nomeado de ilha das complicações. Os fatores de risco são os precursores da erupção do vulcão, alusão aos fatores de risco que provocam as complicações. Logo abaixo da ilha há o desenho um barquinho que está navegando entre os fatores de risco modificáveis e não modificáveis.

O facilitador é responsável por incitar a discussão, esclarecer dúvidas e salientar a importância da mudança de estilo de vida para eliminar os fatores de risco modificáveis, aos quais temos controle. Neste momento foi importante evidenciar que o condutor do barco é a própria pessoa com diabetes e, colocando-o como protagonista do seu próprio cuidado. No mapa, a unidade e equipe de saúde aparecem na margem do rio, com um farol que ilumina o caminho. Sendo assim, foi possível esclarecer que o papel da equipe é cooperar no processo do enfrentamento do diabetes, mas que o caminho é percorrido apenas por eles e que o destino irá depender, em grande parte, das escolhas e mudanças de cada um.

No terceiro encontro abordou-se as temáticas alimentação saudável e atividade física. Para isso foi utilizado o kit de réplicas que continham alimentos in natura/minimamente processados, processados e ultraprocessados (fashion cook). O facilitador pediu para que os participantes agrupassem os alimentos, de acordo com seus conhecimentos nestas três categorias. A maioria dos participantes afirmou consumir excesso de processados e ultraprocessados em suas refeições. Foi relatado consumo regular de minimamente processados, principalmente arroz, feijão e carnes em geral. Alimentos in natura eram raramente consumidos. Relataram que isso ocorre principalmente pela facilidade de aquisição, preço, costume, praticidade no preparo e validade, uma vez que verduras e legumes estragam mais rápido e demandam mais tempo no preparo.

Nesse momento foi salientado que não é proibido consumir nenhum tipo de alimento, mas que a base para uma alimentação saudável demanda aumentar o consumo dos in natura e minimamente processados, utilizando pouco óleo, sal e açúcar no preparo destes. Recomendado a redução gradativa do consumo dos alimentos das outras classes. Nesta etapa, a nutricionista residente ofertou dicas para organizar a rotina alimentar, como comprar as frutas da época que são mais baratas, deixar os alimentos pré-preparados para ganhar tempo e contar com a ajuda da família. Reforçada a importância de não pular refeições e não permanecer longos períodos sem comer, aumentando a frequência e reduzindo as quantidades das refeições (Brasil, 2014).

Em relação a prática de atividades físicas, optou-se por uma dinâmica com o grupo para elucidar alguns aspectos chave. Com o uso de figuras, como de pessoas em academias, caminhando ao ar livre, limpando a casa, indo para o trabalho, entre outras, foi orientado agrupar as imagens em: atividade física ou exercício físico. Após esta etapa e com o auxílio do 
facilitador, foi elucidada as diferenças entre atividade e exercício físico, além de apresentas a recomendação da Org anização Mundial da Saúde de 150 minutos de atividade física/semana (cerca de 20 minutos/ dia), que tem bons resultados na redução das complicações crônicas (Silva, et al., 2018).

Neste tema, introduziu-se a ideia de ser possível atingir bons níveis de atividade física mesmo sem frequentar academias e incluindo hábitos no dia a dia, como optar pelas escadas ao elevador, ir a pé ou de bicicleta ao trabalho, entre outros. Após a dinâmica os participantes perceberam que praticam atividades físicas e que é possível reorganizar a rotina para alcançar os objetivos sem grandes incrementos financeiros e de tempo, aproveitando as próprias atividades de vida diárias.

No quarto encontro a ferramenta disparadora foi o uso de prescrições de duas pessoas para suscitar a discussão sobre terapia medicamentosa. Após listar os medicamentos das prescrições e questionar outros participantes do grupo também utilizavam aqueles medicamentos, de que forma e se sabiam a indicação. Nesta atividade buscou-se a troca de experiência entre o grupo e percepção da semelhança entre os medicamentos utilizados.

Discutiu-se o motivo da insulinização e mitos acerca do tema. A metformina foi o medicamento que mais surgiu em comum no grupo e os integrantes relataram bom conhecimento em relação ao uso, embora tenham surgido relatos de eventos adversos gástricos, diarreia e dificuldade de deglutição pelo tamanho do comprimido, principalmente quando necessário dois comprimidos concomitantemente. Surgiu ainda, em menor proporção, utilização de outros antidiabéticos orais como gliclazida e glibenclamida, além de medicamentos anti-hipertensivos e hipolipemiantes (Brasil, 2014).

Durante as discussões o facilitador elucidou dúvidas, esclareceu divergências e enfatizou a importância da correta utilização dos medicamentos. Discutiu-se as dificuldades da adesão, surgiram aspectos como esquecimento, cansaço, ausência de sintomas e até mesmo falta de confiabilidade no tratamento. Assim, buscou-se elaborar as demandas de forma a ressaltar o papel essencial que a terapia medicamentosa tem no controle do diabetes, e a interdependência com as demais práticas relacionadas para o sucesso do controle da condição.

Em relação às práticas complementares no auxílio do controle do diabetes, os pacientes afirmaram não serem adeptos de nenhuma. No entanto surgiram dúvidas em relação a utilização de suco de Noni (Morinda citrifolia) e chá de folhas de uma planta denominada popularmente de insulina (Cissus sicyoides L). Conforme recomendações da Sociedade Brasileira de Diabetes o uso destas plantas é contraindicado no tratamento do diabetes devido a inconsistência das pesquisas feitas até o momento. Inclusive as publicações científicas sobre o suco de Noni têm trazido muita controvérsia sobre sua segurança como alimento, fato que provocou a proibição da venda de produtos contendo Morinda citrifolia no Brasil (SBD, 2019).

Elucidou-se estes aspectos com os participantes e enfatizou-se a não utilização destes produtos no tratamento do diabetes. Além disso, foi orientado o esclarecimento de dúvidas com os profissionais de saúde. Destacou-se que as práticas integrativas e complementares são um complemento a terapia tradicional do diabetes, pautada em mudança de estilo de vida, alimentação, prática de atividades físicas, medicamentos e saúde mental. As práticas integrativas e complementares mais indicadas no diabetes são: massoterapia de mãos e pés, aromaterapia, musicoterapia e meditação (Feijó, et al., 2012)

No quinto encontro retomaram-se alguns dos temas abordados nos encontros anteriores e foram estabelecidas metas em conjunto, como a redução de $1 \%$ na HbA1c dos com valores inferiores a $8 \%$. No tocante a alimentação, pactuamos o aumento da frequência e diminuição das quantidades das refeições, redução do consumo de alimentos processados e ultraprocessados, aumento da ingestão de alimentos in natura, minimamente processados e aumento do consumo de água.

Também foi estabelecido o aumento gradual de cinco minutos de atividades físicas por dia, até chegar aos 30 minutos diários ou no mínimo 20 minutos/dia, buscando integrar as atividades físicas a rotina. Orientou-se acerca do cuidado diferenciado no uso dos medicamentos e aplicação da insulina diariamente, respeitando os horários, caso haja problema de esquecimento poderá ser utilizado um despertador no celular para auxiliar. Realizar mais atividades prazerosas e de lazer durante a semana, bem como, o envolvimento envolver de familiares e amigos no processo do controle glicêmico. 
Empregou-se um método de avaliação simples para compreender a percepção dos participantes em relação aos atendimentos. Solicitou-se que cada indivíduo falasse uma palavra que representasse o que significou o grupo para ele. As palavras que surgiram foram: gratidão, bom, aprendizado, aproveitável, maravilhoso, ótimo, importante, esclarecedor, especial, união, cuidado, saúde, alegria, comunitário, troca e comunicação.

\section{Considerações Finais}

$\mathrm{O}$ registro adequado das atividades desenvolvidas possibilita a execução de projetos de pesquisa que envolvem o desenvolvimento de práticas clínicas inovadoras. Disto resultam relatos de experiência que podem ser propagados no cotidiano das práticas coletivas na APS, bem como agregar conhecimentos científicos que contribuem para a transformação das ações em saúde, com o aprimoramento do perfil de desfechos clínicos favoráveis. Neste sentindo, este estudo busca contribuir para a ampliação das atividades coletivas, educativas e participativas, por intermédio de ações articuladas e planejadas. Almeja-se promover reflexões sobre a importância da mudança do comportamento e melhoria da qualidade de vida das pessoas com DM2.

\section{Referências}

American Diabetes Association. Diabetes Technology: Standards of Medical Care in Diabetes. (2021). Diabetes Care. (44) 1.

Ancioli, S. (2021). Radicalizar as Práticas de Educação Popular em Saúde. Interface, 25, e200536. 10.1590/Interface.200536

Brasil, Ministério da Saúde, Secretaria de Atenção à Saúde \& Departamento de Atenção Básica. Guia alimentar para a população brasileira. (2014). (2) (1).

Campbell, J. A., Willians, J. S., \& Egede, L. E. (2021). Examining the Relationship Between Delay Discounting, Delay Aversion, Diabetes Self-care Behaviors, and Diabetes Outcomes in U.S. Adults with type 2 diabetes. Diabetes Care, 44(4), 893-900. 10.2337/dc20-2620

Feijó, A. M., Bueno, M. E. N., Ceolin, T., Schwartz, E., Lange, C., \& Meincke, S. M. K. (2012). Plantas medicinais utilizadas por idosos com diagnóstico de diabetes mellitus no tratamento dos sintomas da doença. Rev. Bras. Pl. Med., 14(1), 50-56.

Ferreira Neto, J. L., \& Kind, L. (2010). Práticas grupais como dispositivo na promoção da saúde. Physis: Revista de Saúde Coletiva, 20 (4), 1119-1142. $10.1590 /$ S0103-73312010000400004

Figueiredo, E. N. Estratégia Saúde da Família e Núcleo de Apoio à Saúde da Família: Diretrizes e Fundamentos. (2012).

Fontana, R. T., Flores, F.R., Silva, K. C., Thomas, S. L., Pires, L. G., Oliveira, N. G., \& Feller, S. R. (2020). Reflexões sobre a educação em saúde como um processo emancipatório. Braz. J. Hea. Rev., 3 (3), 5196-5203. 10.34119/bjhrv3n3-096

Gomes, T. F., Silva, A. P. L., Pereira, B. A., \& Rabelo, N, N, F. (2019). Desenvolvimento de um programa de educação em diabetes como ferramenta para a promoção da mudança de hábitos de vida: relato de experiência. Revista Atenas Higeia, 1(1), 31-34.

Instituto Brasileiro de Geografia e Estatística. Pesquisa Nacional de Saúde 2019: percepção do estado de saúde, estilos de vida e doenças crônicas. Brasil, grandes regiões e unidades da federação. (2019).

International Diabetes Federation. (2019). IDF Diabetes Atlas. (9).

Moraes, S.A., Freitas, I. C. M, Gimenos, S. G. A., \& Mondini, L. (2010). Prevalência de diabetes mellitus e identificação de fatores associados em adultos residentes em área urbana de Ribeirão Preto, São Paulo, Brasil, 2006: projeto OBEDIARP. Cad Saúde Pública, 26 (5), 929-941. 10.1590/S0102$311 \mathrm{X} 2010000500015$

Moura, E. C., Gomes, R., \& Pereira, G. M. C. (2017). Percepções sobre a saúde dos homens numa perspectiva relacional de gênero. Ciênc. saúde colet. 22 (1), 291-300. 10.1590/1413-81232017221.17482015

Oliveira, R. E. M., \& Franco, L. J. Glycemic control in elderly people with type 2 diabetes mellitus attending primary health care unit. (2021). Primary Care Diabetes. (15) 4, 733-736. 10.1016/j.pcd.2021.04.011

Secretaria Municipal da Saúde de Ribeirão Preto. (2020). Protocolos e diretrizes de atendimento - Linha de cuidado: hipertensão e diabetes. Programa de atenção às pessoas com doenças crônicas não transmissíveis. Disponível em: https://www.ribeiraopreto.sp.gov.br/files/ssaude/pdf/prot-hip-diab-atendimento1.pdf.

Silva, R. S., Bezerra, J. A. X., Silva, K. V., Silva, N. N., \& Lopes, D. T. 2018. A importância da atividade física em idosos com diabetes. Revista Diálogos em Saúde, (1) 2, 144-158.

Skyler, J. S., Bakris, G. L., Bonifacio, E., Darsow, T., Eckel, R. H., Groop, L., Groop, P. H., Handelsman, Y., Insel, R. A., Mathieu, C., McElvaine, A. T., Palmer, J. P., Pugliese, A., Schatz, D. A., Sosenko, J. M., Wilding, J. P., \& Ratner, R. E. (2017). Differentiation of Diabetes by Pathophysiology, Natural History, and Prognosis. Diabetes, 66(2), 241-255. 10.2337/db16-0806 
Research, Society and Development, v. 10, n. 8, e49910817485, 2021

(CC BY 4.0) | ISSN 2525-3409 | DOI: http://dx.doi.org/10.33448/rsd-v10i8.17485

Sociedade Brasileira de Diabetes. (2019). Diretrizes da Sociedade Brasileira de Diabetes 2019/2020. Disponível em: https://www.diabetes.org.br/profissionais/images/DIRETRIZES-COMPLETA-2019-2020.pdf

Torres, H. C., Pace, A. E., Chaves, F. F., Melendez, G. V., \& Reis, I. A. Avaliação dos efeitos de um programa educativo em diabetes: ensaio clínico randomizado. (2018). Rev. Saúde Pública. (52) 8, 1-11. 10.11606/S1518-8787.2018052007132

Zimmet, P., Alberti, G. K., Magliano, D. J., \& Bennett, P. Diabetes mellitus statistics on prevalence and mortality: facts and fallacies. (2016). Nat Rev Endocrinol., (12) 10, 616-22. 10.1038/nrendo.2016.105 